Görnig, Matthias; Tute, Christian; Liehr, Mario; Lau, Stephan ; Haueisen, Jens;

Figulla, Hans-R.; Leder, Uwe:

\title{
Spatiotemporal correlation analyses: a new procedure for standardisation of DC magnetocardiograms
}

Zuerst erschienen in: Biomedizinische Technik = Biomedical Engineering. - Berlin [u.a.] : de Gruyter. - 51 (2006), 4, p. 198-200.

Erstveröffentlichung: $\quad$ 2006-10-25

ISSN (online): $\quad$ 1862-278X

ISSN (print): $\quad$ 0013-5585

DOI: $\quad$ 10.1515/BMT.2006.036

[Zuletzt gesehen: 43696]

„Im Rahmen der hochschulweiten Open-Access-Strategie für die Zweitveröffentlichung identifiziert durch die Universitätsbibliothek IImenau. “

"Within the academic Open Access Strategy identified for deposition by Ilmenau University Library."

„Dieser Beitrag ist mit Zustimmung des Rechteinhabers aufgrund einer (DFGgeförderten) Allianz- bzw. Nationallizenz frei zugänglich."

"This publication is with permission of the rights owner freely accessible due to an Alliance licence and a national licence (funded by the DFG, German

Research Foundation) respectively." 


\section{Spatiotemporal correlation analyses: a new procedure for standardisation of DC magnetocardiograms}

\author{
Matthias Goernig ${ }^{1, *}$, Christian Tute ${ }^{1}$, Mario \\ Liehr ${ }^{1,2}$, Stephan Lau², Jens Haueisen ${ }^{2,3}$, Hans \\ R. Figulla ${ }^{1}$ and Uwe Leder ${ }^{1}$ \\ 1 Department of Internal Medicine I, Friedrich Schiller \\ University, Jena, Germany \\ 2 Biomagnetic Centre, Department of Neurology, \\ Friedrich Schiller University, Jena, Germany \\ ${ }^{3}$ Institute of Biomedical Engineering and Informatics, \\ University of Technology, IImenau, Germany
}

\begin{abstract}
There is a lack of standard methods for the analysis of magnetocardiograms (MCGs). MCG signals have a shape similar to the ECG ( $P$ wave, QRS complex, $T$ wave). High-quality multichannel recordings can indicate even slight disturbances of de- and repolarisation. The purpose of our study was to apply a new approach in the analysis of signal-averaged DC-MCGs. DC-MCGs (31-channel) were recorded in 182 subjects: 110 patients after myocardial infarction and 72 controls. Spatiotemporal correlation analysis of the QRS complex and T wave patterns throughout the entire heart cycle was used to analyse homogeneity of de- and repolarisation. These plots were compared to standard ECG analyses (electrical axis, Q wave, ST deviation, T polarity and shape). Spatiotemporal correlation analyses seem to be applicable in assessing the course of electrical repolarisation with respect to homogeneity. MCG provided all diagnostic information contained in common ECG recordings at high significance levels. The ECG patterns were included in 5/8 of our parameters for electrical axis, 6/8 for Qwave, $7 / 8$ for ST deviation and $5 / 8$ for T-polarity based on two time series of correlation coefficients. We conclude that our spatiotemporal correlation approach provides a new tool for standardised analysis of cardiac mapping data such as MCG.
\end{abstract}

Keywords: electrocardiography; magnetocardiography; myocardial infarction; repolarization; spatiotemporal analyses.

\section{Introduction}

Whereas several recent studies investigated the clinical significance of magnetocardiography in cardiology [2, 4, 5], no easy and standardised procedure for the interpretation of DC magnetocardiograms (MCGs) has been established so far. In electrophysiology, the spatiotem-

\footnotetext{
*Corresponding author: Matthias Görnig, Department of

Internal Medicine I, Friedrich Schiller University Jena, Erlanger

Allee 101, 07747 Jena, Germany

Phone: + 49-3641-9324101

Fax: +49-3641-9324102

E-mail: matthias.goernig@med.uni-jena.de
}

poral homogeneity of repolarisation contains a great deal of information that is not sufficiently reproduced in electrocardiograms (ECGs). Analysing changes in magnetic field maps over time reconstructed from multichannel MCG data requires complex computer algorithms and may be difficult to interpret.

To overcome these problems in interpretation of MCG data, we applied a spatiotemporal analysis technique that takes advantage of multichannel MCG recordings. In addition, we tested the information content in comparison to typical ECG patterns. With the help of these techniques, we were able to analyse MCG data using simple data-analysis software.

\section{Materials and methods}

A total of 182 subjects were recruited for the study. Of these, 110 were patients (76 males, 34 females; mean age 62 years) mean 15 days (3-64 days) after myocardial infarction (MI). The remaining 72 subjects ( 36 males and 36 females, mean age 46) were healthy controls. All subjects were investigated using signal-averaged DC MCG mapping with a 31-channel biomagnetometer (Philips, Hamburg, Germany) [1]. None of the subjects had ferromagnetic implants. Subjects were lying in a supine position, and continuous data were recorded at a sampling rate of $1000 \mathrm{~Hz}(0-300 \mathrm{~Hz}$ bandwidth). The biomagnetometer was positioned close to the anterior chest wall above the heart and was tilted $20^{\circ}$ to the left.

All recordings lasted for $10 \mathrm{~min}$ for each patient. Baseline drift artefacts in the DC recordings due to interference from external electromagnetic fields were minimised by active and passive magnetic shielding (Ak3b; Vacuumschmelze, Hanau, Germany) of the biomagnetometer. Active shielding was achieved by continuous measurement and adjustment of the signal of a reference magnetometer inside the room to zero using large external compensation coils [6].

To remove $50-\mathrm{Hz}$ power line interference from the DC MCG data, 21-sample Gaussian-weighted window averaging was performed. The raw data were averaged using QRS maximum coherence matching (MCM) with a template beat [3]. We used the mean global field power (MGFP) of the signal, resulting in an average heartbeat for each subject. Onsets, offsets and maxima of the QRS complex and $T$ wave were measured by eye. Heart cycles preceded or followed by premature beats were excluded from averaging.

We computed eight parameters for two time series of linear correlation coefficients. The correlation coefficients were calculated by covariance over the product of the related standard deviation:

$\operatorname{corr}_{X, Y}=\operatorname{cov}(X, Y) / \operatorname{var}(X) \cdot \operatorname{var}(Y)$.

Series I was the correlation of the signal at the QRS max- 
imum for each single time point. Series II was the correlation of the signal at the $\mathrm{T}$ maximum for each single time point. For both of these time series, the mean value and standard deviation of the calculated correlation values at the time interval QRS maximum minus 20 ms until QRS maximum plus $20 \mathrm{~ms}$, and at the time interval T maximum minus $20 \mathrm{~ms}$ until $\mathrm{T}$ maximum plus $20 \mathrm{~ms}$, were calculated. This resulted in eight parameters:

- Mean corr QRS at QRS,

- STDEV corr QRS at QRS,

- Mean corr QRS at T,

- STDEV QRS at T,

- Mean corr T at T,

- STDEV corr T at T,

- Mean corr T at QRS, and

- STDEV corr T at QRS.

Figure 1 shows time series I, with correlation of QRS maximum at each single time point of the MGFP for a patient after $\mathrm{Ml}$ and a healthy volunteer.

The spatiotemporal plots were compared to standard ECG patterns (discrete variables): electrical axis, normal axis, left axis deviation, right axis deviation, indeterminate axis deviation, $Q$ wave Einthoven and Wilson, ST deviation Einthoven and Wilson, and T polarity Einthoven. A two-tailed t-test for independent samples (significance level $p<0.05$ ) and Pearson's correlation (significance level $p<0.01$ ) were applied.

\section{Results}

The maximum baseline drift during the reference recordings was below 10 pT in all measurements (i.e., below $0.016 \mathrm{pT} / \mathrm{s}$ ). In recordings for all subjects, respiratory baseline fluctuations were below 5 pT. The systematic drift of the baseline that was not compensated by active shielding of the DC MCG device was below $10 \mathrm{pT} / 10$ min. Table 1 shows ECG parameters significantly correlated with MCG parameters.
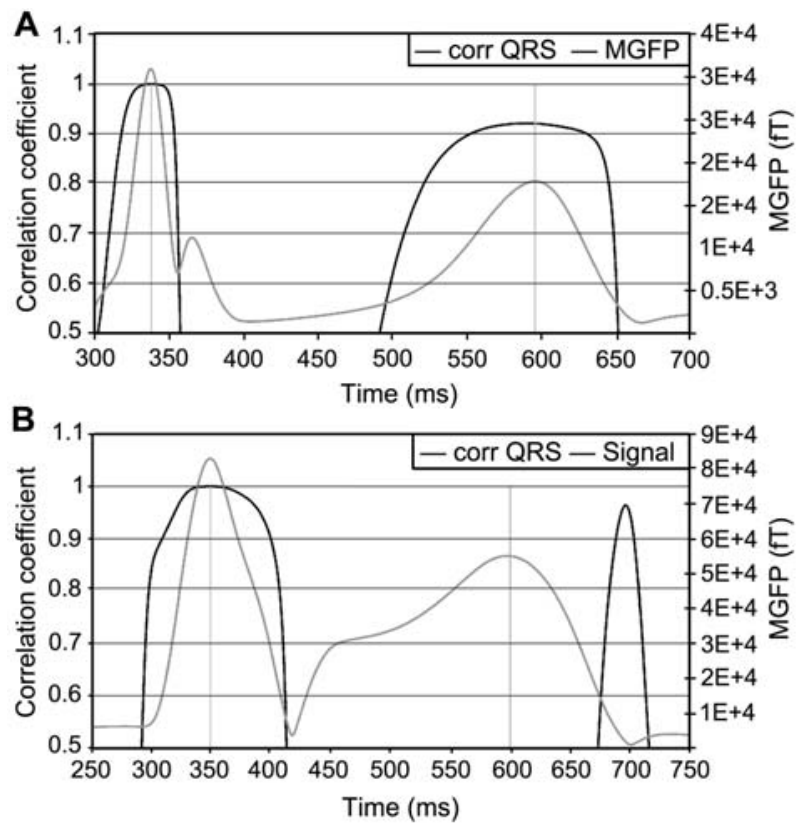

Figure 1 Spatial correlation analysis of QRS maximum throughout QRS and T interval of the averaged MGFP signal of 31-channel DC-MCGs in (A) a healthy 59-year-old female and (B) a 36-year-old male 6 days after myocardial infarction. Left ordinates correspond to the correlation values, right ordinates (in fT) correspond to MGFP of the signal.

\section{Discussion}

In general, two different approaches are used to analyse MCG data: looking at averaged DC signals, which have a shape similar to standard ECGs, or solving the inverse problem. Inverse solutions on magnetic field maps require complex computation and may be difficult to interpret because of unstable solutions. In contrast to the common practice of applying high pass filtering when recording the MCG, we used DC MCGs to detect verylow-frequency components of the STU segment, which

Table 1 Significance levels for comparison of 12-lead ECG parameters and spatiotemporal analysis of DC MCGs in 182 subjects (110 after myocardial infarction, 72 controls).

\begin{tabular}{|c|c|c|c|c|c|c|c|}
\hline \multirow[t]{2}{*}{ MCG $(n=182)$} & & \multicolumn{6}{|l|}{ ECG } \\
\hline & & $\begin{array}{l}\text { Electrical } \\
\text { axis }\end{array}$ & $\begin{array}{l}\text { Q wave } \\
\text { Einthoven }\end{array}$ & $\begin{array}{l}\text { Q wave } \\
\text { Wilson }\end{array}$ & $\begin{array}{l}\text { ST deviation } \\
\text { Einthoven }\end{array}$ & $\begin{array}{l}\text { ST deviation } \\
\text { Wilson }\end{array}$ & $\begin{array}{l}\mathrm{T} \text { polarity } \\
\text { Einthoven }\end{array}$ \\
\hline \multirow[t]{2}{*}{ Mean corr QRS at QRS } & Two-tailed t-test & 0.201 & 0.710 & 0.14 & 0.14 & 0.974 & $0.031^{*}$ \\
\hline & Pearson's correlation & 0.950 & 0.028 & 0.181 & $0.001^{\star \star}$ & $0.002^{\star \star}$ & 0.160 \\
\hline \multirow[t]{2}{*}{ STDEV corr QRS at QRS } & Two-tailed t-test & $0.010^{*}$ & 0.461 & $0.006^{\star}$ & 0.310 & $0.032^{*}$ & 0.078 \\
\hline & Pearson's correlation & 0.190 & 0.55 & 0.205 & 0.067 & 0.195 & 0.131 \\
\hline \multirow[t]{2}{*}{ Mean corr $\mathrm{T}$ at $\mathrm{T}$} & Two-tailed t-test & 0.446 & 0.715 & 0.066 & 0.848 & 0.688 & 0.075 \\
\hline & Pearson's correlation & 0.057 & 0.027 & 0.137 & 0.014 & 0.030 & 0.132 \\
\hline \multirow[t]{2}{*}{ STDEV corr $\mathrm{T}$ at $\mathrm{T}$} & Two-tailed t-test & 0.055 & 0.062 & 0.198 & $0.040^{\star}$ & 0.696 & 0.222 \\
\hline & Pearson's correlation & 0.143 & 0.139 & 0.098 & 0.152 & 0.290 & 0.091 \\
\hline \multirow[t]{2}{*}{ Mean corr QRS at $T$} & Two-tailed t-test & $<0.001^{*}$ & 0.054 & $<0.001^{\star}$ & 0.956 & $<0.001^{*}$ & $<0.001^{*}$ \\
\hline & Pearson's correlation & 0.352 & 0.143 & 0.330 & $0.004^{\star \star}$ & 0.298 & 0.361 \\
\hline \multirow[t]{2}{*}{ STDEV corr QRS at T } & Two-tailed t-test & $<0.001^{*}$ & $0.017^{\star}$ & $0.030^{\star}$ & 0.052 & 0.262 & $0.006^{\star}$ \\
\hline & Pearson's correlation & 0.267 & 0.176 & 0.161 & 0.011 & 0.840 & 0.203 \\
\hline \multirow[t]{2}{*}{ Mean corr T at QRS } & Two-tailed t-test & $0.003^{*}$ & 0.052 & $<0.001^{*}$ & 0.998 & $<0.001^{*}$ & $<0.001^{\star}$ \\
\hline & Pearson's correlation & 0.350 & 0.144 & 0.373 & $<0.001^{\star \star}$ & 0.302 & 0.361 \\
\hline \multirow[t]{2}{*}{ STDEV corr T at QRS } & Two-tailed t-test & $<0.001^{\star}$ & 0.051 & $0.004^{*}$ & 0.328 & 0.176 & $0.004^{*}$ \\
\hline & Pearson's correlation & 0.222 & 0.145 & 0.210 & 0.073 & 0.101 & 0.213 \\
\hline
\end{tabular}

${ }^{*} p<0.05$.

${ }^{* *} p<0.01$. 
can only be detected without a low frequency limit, especially for the course of ST- and TU-segment transitions, as well as $U$-wave duration. Our spatiotemporal analysis technique can easily be used with standard data analysis software and is open to visual interpretation.

Our results have several implications. In a first step we could show that relevant information from the standard ECG was included in selected patterns of our spatiotemporal plots for healthy subjects and MI patients. Because of the extended field of view, recording of vector gradients and analyses without a lower cut-off frequency for multichannel MCG, as well as the high signal to noise ratio (SNR) for magnetic signals, we expect that more information on pathological conditions can be obtained.

For the next step we plan to identify disturbances of de- and repolarisation in patients presenting with nondiagnostic 12-lead ECG but with abnormalities in coronary perfusion or pumping function of the left ventricle at rest and under stress conditions. Another application may involve the monitoring of antiarrhythmic drug effects that interfere with intrinsic repolarisation properties. The low specificity of parameters of de- and repolarisation disturbances has to be considered analytically.

\section{Conclusion}

We applied spatiotemporal analyses of DC MCGs and were able to show abnormal findings of the ECG accom- panied by significant changes in time series of linear correlation coefficients. Owing to the additional information provided by spatiotemporal MCGs, this approach can be used to detect pathological de- and repolarisation patterns that are missed in 12-lead ECGs.

\section{References}

[1] Dössel O, David B, Fuchs $M$, Krüger J, Kullmann WH, Ludeke KM. A modular approach to multichannel magnetometry. Clin Phys Physiol Meas 1991: 12 (Suppl B): 75-79.

[2] Fenici R, Brisinda D, Meloni AM. Clinical application of magnetocardiography. Expert Rev Mol Diagn 2005; 5: 291-313.

[3] Huck M, Haueisen J, Hoenecke O. QRS amplitude and shape variability in magnetocardiograms. PACE 2000; 23 : 234-242.

[4] Koskinen R, Lehto M, Vaanana H, et al. Measurement and reproducibility of magnetocardiographic filtered atrial signal in patients with paroxysmal lone atrial fibrillation and in healthy subjects. J Electrocardiol 2005; 38: 330-336.

[5] Park JW, Hill PM, Chung N, Hugenholz PG, Jung F. Magnetocardiography predicts coronary artery disease in patients with acute chest pain. Ann Noninvas Electrocardiol 2005; 10: 312-323.

[6] Platzek D, Nowak H, Gießler F, Röther J, Eiselt M. Active shielding to reduce low frequency disturbances in direct current near biomagnetic measurements. Rev Sci Instrum 1999; 70: 2465-2470. 